\title{
Modulation of the circadian clock by glucocorticoid receptor in $H 295 R$ cell line
}

\author{
Zsolt Nagy ${ }^{1,2}$, Henriett Butz ${ }^{3}$, Istvan Liko², Karoly Racz ${ }^{1,3}$, Attila Patocs ${ }^{2,4}$ \\ 12nd Department of Medicine, Faculty of Medicine, Semmelweis University, Budapest, \\ Hungary \\ ${ }^{2}$ Hungarian Academy of Sciences-Semmelweis University "Lendulet" Hereditary \\ Endocrine Tumors Research Group, Budapest, Hungary \\ ${ }^{3}$ Hungarian Academy of Sciences-Semmelweis University Molecular Medicine Research \\ Group, Budapest, Hungary \\ ${ }^{4}$ Department of Laboratory Medicine Institute, Faculty of Medicine, Semmelweis \\ University, Budapest, Hungary
}

Background: Peripheral clocks are set by different nervous, hormonal and metabolic stimuli and regulate the circadian expression of several genes. It has been demonstrated that circadian oscillation of gene expression can be detected in various cell lines in vitro.

Aim: To explore whether a peripheral clock could be induced in human adrenocortical cell line H295R and what are the effects of glucocorticoids on this clock system.

Interactive feed-back loops of the molecular circadian clock machinery Rhythmic expression of clock genes in H295R ell line upon serum shock treatment

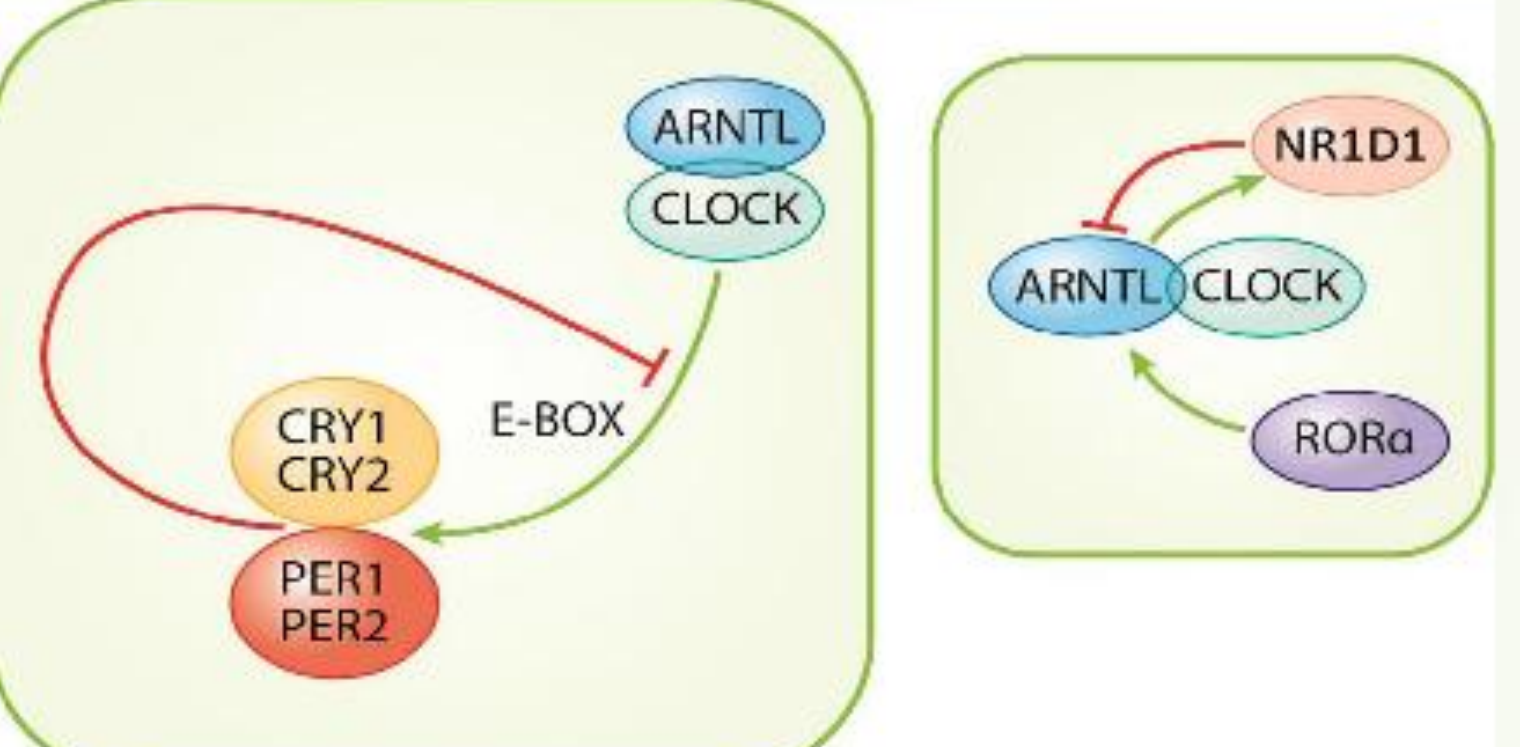
shock induced clock genes

hours

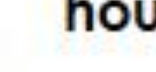

Dex modifies the pattern of serum
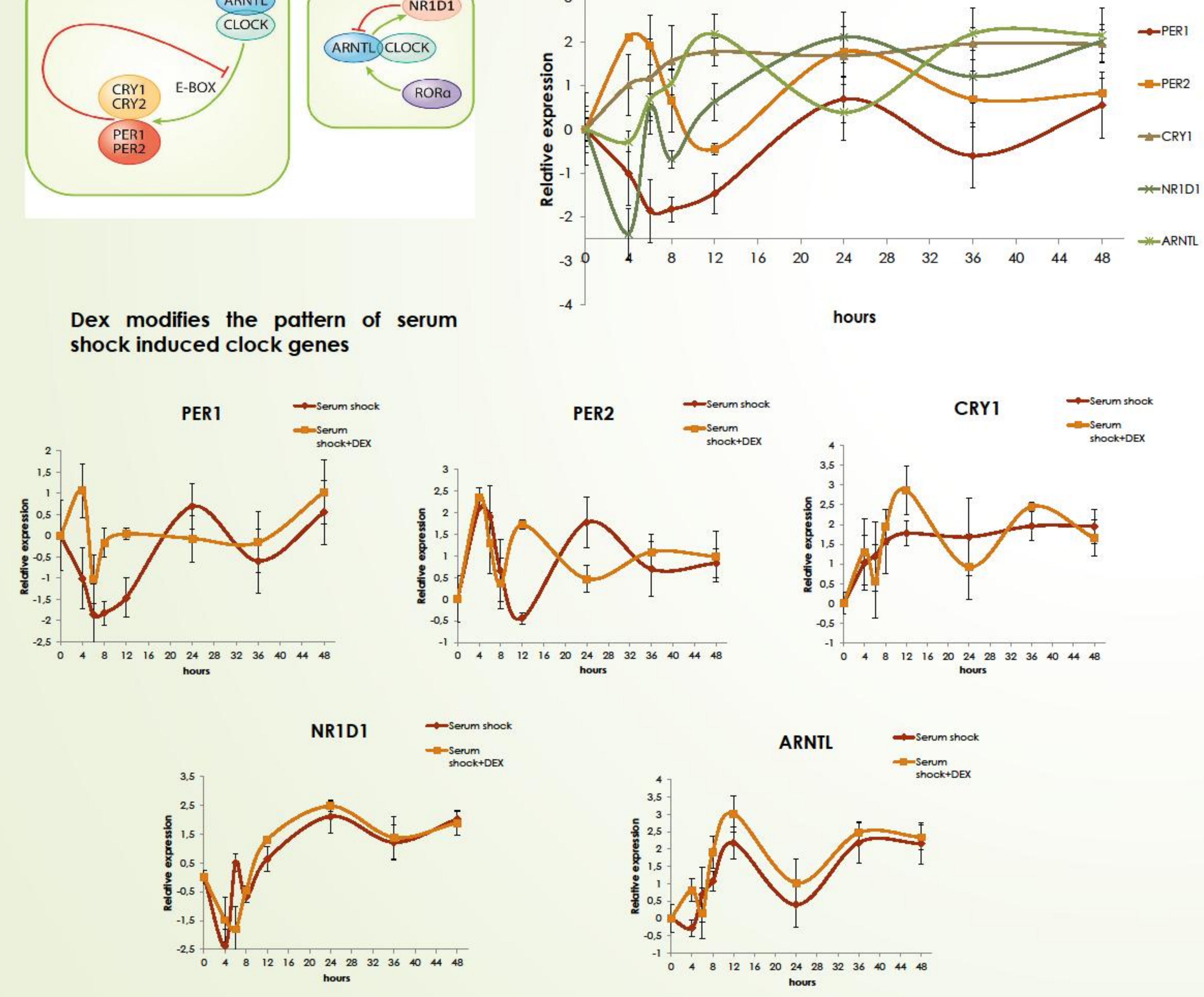

1

Dex induces the expression of PER1, PER2, CRY1 and suppresses NRID1 in

glucocortiocid receptor dependent manner in $\mathrm{H} 295 \mathrm{R}$ cell line
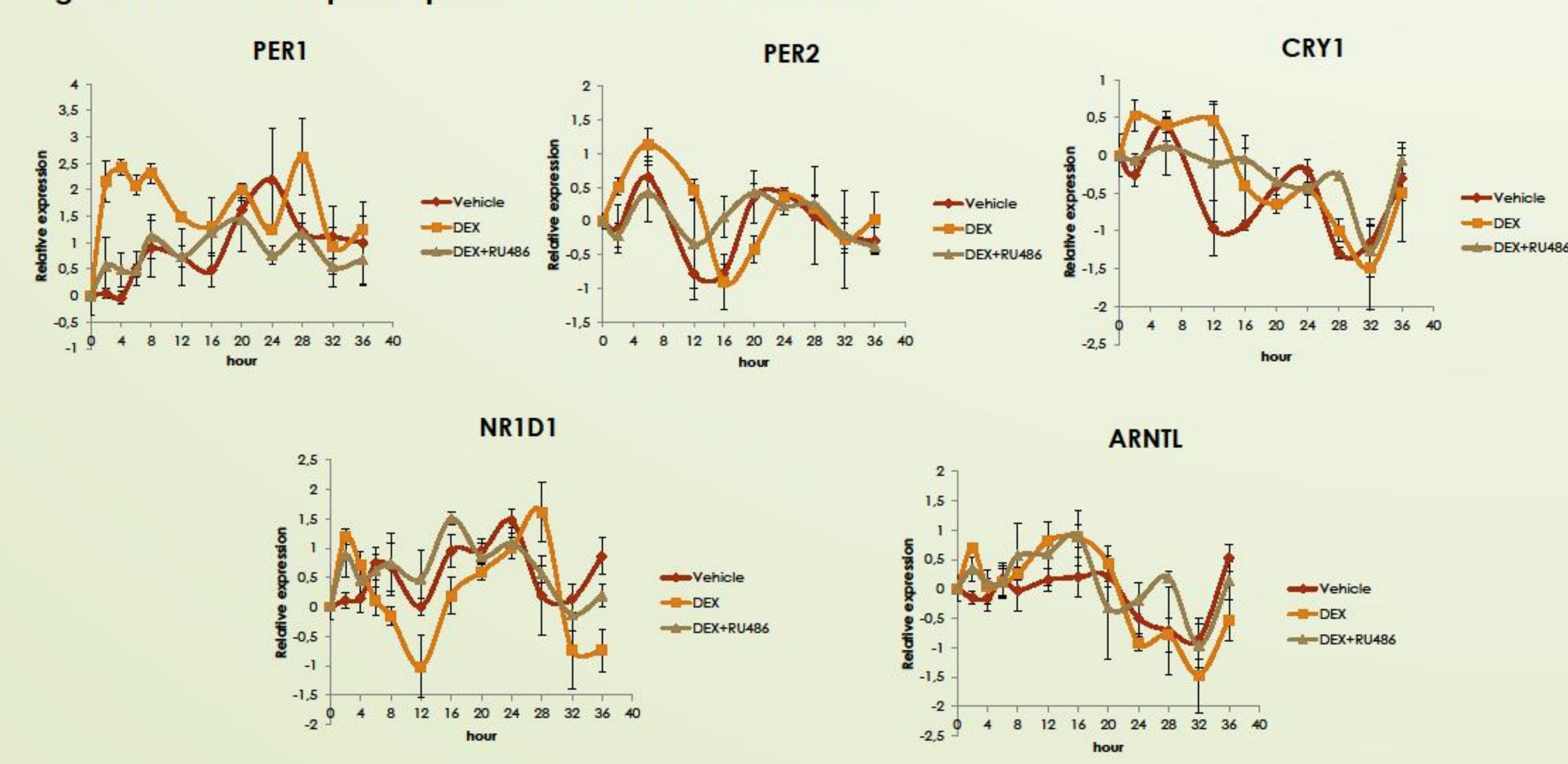

Methods:

H295R human adrenocortical cell line was studied.

- For serum shock experiments cells were serum starved for $24 \mathrm{~h}$ and incubated with $30 \% \mathrm{Nu}$ Serum for $2 \mathrm{~h}$ then returned to normal medium with either $0.04 \%$ ethanol or $100 \mathrm{nmol}$ dexamethasone (Dex).

- For Dex experiments cells were serum starved for $24 \mathrm{~h}$, maintained in serum free medium and treated with $0.04 \%$ ethanol or $100 \mathrm{nmol}$ Dex with or without $1 \mu \mathrm{mol}$ RU486.

- Cells were harvested at the indicated time points. All experiments were carried out in triplicate.

- Total RNA was isolated with miRNeasy Mini Kit (Qiagen) and transcribed with Invitrogen Superscript VILO reverse transcriptase (LifeTechnologies).

- RT-PCR was carried out using predesigned TaqMan Gene Expression Assays. PER1, PER2, CRY1, ARNTL, NR1D1, NR3C1, StAR, POMC, CRH and actin expressions were measured by qRT-PCR on 7500 Fast Real-Time PCR system

\section{Results:}

- After synchronization of cells the rhythmic oscillation of clock genes PER1, PER2, NR1D1, and ARNTL was observed.

- Glucocorticoid treatment induced a rapid respond of PER1 in a glucocorticoid receptor (GR)-dependent manner. Continuous glucocorticoid stimulation caused elevation of PER1 and altered its rhythm.

- Glucocorticoid treatment induced the expression of PER2 and delayed its phase, increased expression of CRY1 and, after 6 hours, it suppressed expression of NR1D1.

- Administration of a glucocorticoid receptor antagonist, RU486 disrupted the circadian oscillation of clock genes and prevented the acute changes in PER1, PER2 and CRY1 levels.

- These alterations occurred independently from ACTH or $\mathrm{CRH}$.

Conclusions: Our data demonstrated that a peripheral clock system is present in human adrenocortical cell line and periodic oscillation of clock genes are influenced by glucocorticoids.

\section{Acknowledgement:}

Hungarian Scientific Research Fund (OTKA, PD100648 (AP)) and Research and Technology Innovation Fund, National Developmental Agency (KTIA-AIK-2012-12-1-0010). 